\title{
Evaluation of preoperative risk factors for postpancreatectomy hemorrhage
}

\author{
Wataru Izumo $^{1}$ (D) $\cdot$ Ryota Higuchi $^{1}$ (D) $\cdot$ Takehisa Yazawa $^{1} \cdot$ Shuichiro Uemura $^{1} \cdot$ Masahiro Shiihara $^{1} \cdot$ Masakazu Yamamoto $^{1}$
}

Received: 5 July 2019 / Accepted: 27 September 2019 / Published online: 24 October 2019

(C) The Author(s) 2019

\begin{abstract}
Purpose To investigate the risk factors for post-pancreatectomy hemorrhage (PPH).

Methods The incidence, outcome, and risk factors for PPH were evaluated in 1169 patients who underwent pancreatectomy.

Results The incidence and mortality rates of PPH were 3\% and $11 \%$ in all pancreatectomies, $4 \%$ and $11 \%$ in pancreatoduodenectomy, $1 \%$ and $20 \%$ in distal pancreatectomy, and $3 \%$ and $0 \%$ in total pancreatectomy, respectively. Male sex [odds ratio (OR) 2.32], body mass index (BMI) $\geq 25 \mathrm{~kg} / \mathrm{m}^{2}$ (OR 3.70), absence of diabetes mellitus (DM; HbA1c $\leq 6.2 \%$; OR 3.62), and pancreatoduodenectomy (OR 3.06) were risk factors for PPH after all pancreatectomies. The PPH incidence was $0 \%$, $1 \%, 2 \%, 6 \%$, and $20 \%$ in patients with risk scores of $0(n=65), 1(n=325), 2(n=455), 3(n=299)$, and $4(n=25)$, respectively. The differences between risk-score groups $0-2(2 \%)$ and 3-4 (7\%) were significant $(P<0.05$, OR 4.7). In patients who had undergone pancreatoduodenectomy, postoperative pancreatic fistula (POPF; OR 31.7) and absence of DM (OR 3.45) were risk factors for PPH. There was no significant association between POPF and PPH after distal pancreatectomy $(P=0.28)$. The incidence of POPF post-pancreatoduodenectomy was $20 \%$. BMI $\geq 25 \mathrm{~kg} / \mathrm{m}^{2}$ (OR 3.17), serum albumin $<3.5 \mathrm{~g} / \mathrm{dl}(\mathrm{OR} 1.77$ ), absence of DM (OR 1.75), distal extrahepatic bile duct carcinoma (OR 4.05), and carcinoma of the papilla of Vater (OR 5.19) were risk factors for POPF post-pancreatoduodenectomy.

Conclusion Our study clarified the preoperative risk factors for PPH and recommends using a risk scoring system that includes "absence of DM" for predicting PPH.
\end{abstract}

Keywords Post-pancreatectomy hemorrhage $\cdot$ Preoperative $\cdot$ Risk factor $\cdot$ Pancreatic fistula $\cdot$ Pancreatoduodenectomy

\section{Introduction}

Recently, the surgical technique for pancreatectomy has improved; however, pancreatectomy is associated with many complications postsurgery [1-4]. Although the frequency of postpancreatectomy hemorrhage (PPH) is not high, it results in severe adverse outcomes. The incidence rate of PPH and the mortality rate following PPH have been reported to be 3-

Electronic supplementary material The online version of this article (https://doi.org/10.1007/s00423-019-01830-w) contains supplementary material, which is available to authorized users.

Ryota Higuchi

higuchi.ryota@twmu.ac.jp

1 Department of Surgery, Institute of Gastroenterology, Tokyo Woman's Medical University, 8-1 Kawada-cho, Shinjuku-ku, Tokyo 162-8666, Japan
$16 \%$ and $16-36 \%$, respectively [4-12]. The International Study Group on Pancreatic Surgery (ISGPS) defined PPH in 2007 [13]. Some studies have reported that postoperative pancreatic fistula (POPF), postoperative bile leakage, and postoperative abdominal infection are risk factors for PPH [5-12]. Although this information is useful, it includes intraoperative and postoperative factors that can only be known after surgery. Other studies reported that male sex, high body mass index (BMI), and low serum albumin (Alb) levels, which are preoperative factors, are associated with PPH [8, 10, 12, 14]. However, the preoperative risk factors for PPH have not been fully elucidated yet. Therefore, the aim of this study was to clarify the preoperative risk factors for $\mathrm{PPH}$.

\section{Material and methods}

Between 2005 and 2018, 1169 patients underwent pancreatectomy at the Department of Surgery, Institute of Gastroenterology, 
Tokyo Woman's Medical University. Parameters that were evaluated included the incidence, outcomes, time of onset, location, severity, grade, and risk factors of PPH. Clinical data that were collected and examined included age; sex; preoperative BMI; white blood cell (WBC) count; serum levels of Alb, C-reactive protein (CRP), and hemoglobin A1c (HbA1c); preoperative drainage for obstructive jaundice; preoperative treatment (chemotherapy or chemoradiotherapy); operative procedure, duration of surgery; amount of intraoperative blood loss; vascular resection; pancreatojejunostomy (PJ) anastomosis method; falciform ligament wrapping of the gastroduodenal artery (GDA) stump; clipping of the GDA stump; PJ stent; pathological tissue type; pancreatic fistula; delayed gastric emptying (DGE); and postoperative bile leakage. The significant factors identified by multivariate analysis to cause $\mathrm{PPH}$ were defined as risk factors for PPH. The risk score was calculated, with 1 point assigned to each risk factor, and the incidence of PPH was examined for each risk score number.

The patients' characteristics for all pancreatectomies are shown in Supplemental Table 1. Six hundred fifty-five patients $(56 \%)$ were men; the median preoperative BMI level was $21.5 \mathrm{~kg} / \mathrm{m}^{2}$ (range $14.3-36.8 \mathrm{~kg} / \mathrm{m}^{2}$ ); 421 patients $(36 \%)$ had diabetes mellitus (DM); 675 patients $(57 \%)$ underwent pancreatoduodenectomy (PD); 211 patients (18\%) had POPF grade B (15.6\%, 182 patients) or C $(2.5 \%$, 29 patients); 279 (24\%) patients underwent HDL wrapping of the GDA stump; none of the patients with omental grafts underwent GDA stump wrapping, and all patients underwent vascularized graft placement of the GDA stump using the falciform ligament; 26 (2\%) patients underwent clipping of the GDA stump.

\section{Definitions}

\section{PPH, POPF, and DGE}

The PPH, POPF, and DGE grades were defined using ISGPS guidelines $[13,15,16]$ and categorized into 3 (grades A, B, and $\mathrm{C}$ ). Since the grade $\mathrm{A}$ cases were not clinically relevant and not a target for treatment, we only considered grade $\mathrm{B}$ or $\mathrm{C}$ cases in this study. Patients with grades B or C PPH were at risk for death, and blood transfusion, angiography, embolization, relaparotomy, and intensive treatment in the intensive care unit were needed to improve the condition of these patients.

\section{Diabetes mellitus}

HbA1c level $\geq 6.3 \%$ was defined as DM in this study because the normal value of HbA1c in Japan is determined to be $\leq 6.2 \%$.

\section{Surgical policy}

Pancreatectomy with typical lymph node dissection for carcinoma involves regional lymph node dissection, and partial lymph node dissection was performed for inflammation and low malignant tumors. Regional lymph node dissection involves excision of the plexus in the right half of the SMA and dissection of the hepatoduodenal ligament from the left and right hepatic artery bifurcation to the upper margin of the pancreas. The reconstruction method was the Child's procedure. All patients underwent PJ anastomosis for gastroenterological reconstruction, and no patient underwent pancreatogastrostomy. Usually, the GDA stump was double ligated, and beginning in 2018, additional clipping (Hem-o-lok clip®; Telefrex Medical, USA) was performed on the GDA stump. Moreover, beginning in 2010, the GDA stump was wrapped with the falciform ligament. We performed duct-to-mucosa anastomosis for PJ until 2016 and modified Blumgart anastomosis after 2017. The surgeon chose PJ stenting if the diameter of the PJ anastomosis was narrow, reconstruction was difficult, and the remaining pancreas was soft.

\section{Mortality rate}

The rate of inpatient death related to PPH during hospitalization was used as the mortality rate in this study.

\section{Statistical analysis}

Univariate and multivariate logistic regression analyses were performed to determine independent predictors of PPH in patients who had undergone pancreatectomy. Only factors that were significant on univariate analysis were subjected to multivariate analysis. A $P$ value $<0.05$ was considered statistically significant. All the analyses were performed using JMP version 12.1.9 for Windows (SAS Institute Inc., Cary, NC, USA).

\section{Results}

\section{Incidence, mortality, classification, reason, and treatment of PPH after all pancreatectomies (Table 1}

The incidence of PPH grades B or C was 3\% in all 1169 patients undergoing pancreatectomy, $4 \%$ in the 675 patients undergoing $\mathrm{PD}, 1 \%$ in the 406 patients undergoing distal pancreatectomy (DP), and $3 \%$ in the 88 patients undergoing total pancreatectomy (TP). The incidence of late onset time, extraluminal location, high severity, bleeding from pseudoaneurysm, and interventional radiology 
Table 1 Incidence, mortality, classification, reason, and treatment of post-pancreatectomy hemorrhage grades B or C after all pancreatectomy

\begin{tabular}{|c|c|c|c|c|c|}
\hline Category & Definition & All & PD & DP & $\mathrm{TP}$ \\
\hline Total number of PPHs & & $n=35$ & $n=27$ & $n=5$ & $n=3$ \\
\hline Incidence of PPH & & $3 \%(35 / 1169)$ & $4 \%(27 / 675)$ & $1 \%(5 / 406)$ & $3 \%(3 / 88)$ \\
\hline $\begin{array}{l}\text { Mortality among patients } \\
\text { with PPH }\end{array}$ & & $4(11 \%)$ & $3(11 \%)$ & $1(20 \%)$ & $0(0 \%)$ \\
\hline $\begin{array}{l}\text { Median onset time } \\
\text { from initial surgery } \\
\text { (days), (range) }\end{array}$ & & $22(0-65)$ & $24(0-47)$ & $9(0-27)$ & $2(0-65)$ \\
\hline Time of onset & Early/late & $4(4 \%) / 31(96 \%)$ & $1(4 \%) / 26(96 \%)$ & $1(20 \%) / 4(80 \%)$ & $2(67 \%) / 1(33 \%)$ \\
\hline Location & Intraluminal/extraluminal & $9(26 \%) / 26(74 \%)$ & $7(26 \%) / 20(74 \%)$ & $1(20 \%) / 4(80 \%)$ & $1(33 \%) / 2(67 \%)$ \\
\hline Severity & Mild/severe & $9(19 \%) / 26(81 \%)$ & $5(19 \%) / 22(81 \%)$ & $2(40 \%) / 3(60 \%)$ & $2(67 \%) / 1(33 \%)$ \\
\hline \multirow[t]{2}{*}{ Reason } & $\begin{array}{l}\text { Pseudoaneurysm } \\
\text { GDA/HA/SMA/SPA } \\
\text { Bleeding from the stump of the } \\
\text { pancreas, greater omentum } \\
\text { or retroperitoneal }\end{array}$ & $\begin{array}{l}22(66 \%) \\
13 / 5 / 1 / 3 \\
7(19 \%)\end{array}$ & $\begin{array}{l}18(66 \%) \\
12 / 5 / 1 / 0 \\
5(19 \%)\end{array}$ & $\begin{array}{l}3(60 \%) \\
1 / 0 / 0 / 2 \\
1(20 \%)\end{array}$ & $\begin{array}{l}1(33 \%) \\
0 / 0 / 0 / 1 \\
1(33 \%)\end{array}$ \\
\hline & Gastrointestinal bleeding & $6(15 \%)$ & $4(15 \%)$ & $1(20 \%)$ & $1(33 \%)$ \\
\hline Treatment & $\begin{array}{l}\text { Interventional radiology } \\
\text { Conservative treatment with } \\
\quad \text { blood transfusion } \\
\text { Endoscopic treatment } \\
\text { Surgery } \\
\text { Interventional radiology } \\
\text { plus surgery }\end{array}$ & $\begin{array}{l}19(63 \%) \\
6(15 \%) \\
5(11 \%) \\
4(7 \%) \\
1(4 \%)\end{array}$ & $\begin{array}{l}17(63 \%) \\
4(15 \%) \\
3(11 \%) \\
2(7 \%) \\
1(4 \%)\end{array}$ & $\begin{array}{l}2(40 \%) \\
1(20 \%) \\
1(20 \%) \\
1(20 \%) \\
0(0 \%)\end{array}$ & $\begin{array}{l}0(0 \%) \\
1(33 \%) \\
1(33 \%) \\
1(0 \%) \\
0(0 \%)\end{array}$ \\
\hline
\end{tabular}

$P P H$ postpancreatectomy hemorrhage, GDA gastroduodenal artery, $H A$ hepatic artery, SMA superior mesenteric artery, SPA splenic artery, $P D$ pancreatoduodenectomy, $D P$ distal pancreatectomy, $T P$ total pancreatectomy

Classification categories were defined by the International Study Group of Pancreatic Surgery [7]

Time of onset: early hemorrhage means that bleeding occurred $\leq 24 \mathrm{~h}$ after the end of the operation; late hemorrhage means that bleeding occurred $>24 \mathrm{~h}$ after the end of the index operation

Location: intraluminal hemorrhage means bleeding from the gastrointestinal tract; extraluminal hemorrhage means bleeding from the abdominal cavity Severity: Mild means that the patient's condition was not severe enough to necessitate invasive treatment; severe means that the patient's condition was poor, and it was necessary to perform intensive treatment

treatment were $96 \%, 74 \%, 81 \%, 66 \%$, and $63 \%$, respectively. The median onset time of all PPH and bleeding from pseudoaneurysms from the initial surgery was 22 and 21 days, respectively. Mortality among patients with PPH was $11 \%$. These patients died from multiple organ dysfunction caused by bleeding from pseudoaneurysms. The overall mortality rate within 30 and 90 days was $0.3 \%$ (3/1169) and $0.6 \%$ (7/1169), respectively. Mortality from PPH within 30 and 90 days was $0.2 \%(2 / 1169)$ and $0.3 \%$ (3/1169), respectively.

\section{Univariate and multivariate analyses of risk factors for PPH after all pancreatectomies (Table 2) and PPH rate by independent risk factors (Supplemental Table 2)}

In multivariate analysis, male sex (odds ratio [OR] 2.32), BMI $\geq 25 \mathrm{~kg} / \mathrm{m}^{2}$ (OR 3.70), absence of DM (OR 3.62), and PD (OR 3.06) were significant independent risk factors for PPH in all patients undergoing pancreatectomy $(P<0.05)$. When assessing using these risk factors, the incidence of PPH after all pancreatectomies in patients with a risk score of $0(n=65)$, $1(n=325), 2(n=455), 3(n=299)$, and $4(n=25)$ was $0 \%$, $1 \%, 2 \%, 6 \%$, and $20 \%(P<0.0001)$, respectively (Supplemental Table 2). The differences between risk-score groups 0-2 (2\%) and 3-4 (7\%) were significant $(P<0.05$, OR 4.7).

\section{Univariate and multivariate analyses of risk factors for PPH after PD (Table 3)}

Because PD was risk factor of PPH after all pancreatectomies, we evaluated risk factors for $\mathrm{PPH}$ after PD, excluding DP and TP. In multivariate analysis, POPF (OR 31.7) and absence of DM (OR 3.45) were independent risk factors for PPH after PD $(P<0.05)$. There was no significant difference in the incidence of PPH between patients with and without falciform ligament wrapping of the GDA stump [5.7\% (11/276) and 2.8\% (16/399), respectively; $P=0.059$ ]. The incidence of POPF after PD was $20 \%$. 
Table 2 Univariate and multivariate analyses of risk factors for post-pancreatectomy hemorrhage grades B or C after all pancreatectomy

\begin{tabular}{|c|c|c|c|c|c|c|c|}
\hline \multirow[b]{2}{*}{ Risk factors } & \multirow[b]{2}{*}{ Definition } & \multirow[b]{2}{*}{$n$} & \multicolumn{2}{|l|}{ Univariate } & \multirow[b]{2}{*}{$P$ value } & \multicolumn{2}{|l|}{ Multivariate } \\
\hline & & & PPH & $\begin{array}{l}\text { OR } \\
(95 \% \mathrm{CI})\end{array}$ & & $\begin{array}{l}\text { OR } \\
(95 \% \mathrm{CI})\end{array}$ & $P$ value \\
\hline \multirow[t]{2}{*}{ Age (years) } & $<65$ & 493 & $12(2.4 \%)$ & 1 & 0.34 & & \\
\hline & $\geq 65$ & 676 & $23(3.4 \%)$ & $1.41(0.70-2.87)$ & & & \\
\hline \multirow[t]{2}{*}{ Sex } & Female & 518 & $8(1.5 \%)$ & 1 & 0.0013 & 1 & 0.034 \\
\hline & Male & 651 & $27(4.2 \%)$ & $2.76(1.24-6.12)$ & & $2.32(1.02-5.26)$ & \\
\hline \multirow[t]{2}{*}{ BMI $\left(\mathrm{kg} / \mathrm{m}^{2}\right)$} & $<25$ & 1011 & $24(2.4 \%)$ & 1 & 0.0027 & 1 & 0.0009 \\
\hline & $\geq 25$ & 158 & $11(7.0 \%)$ & $3.08(1.48-6.41)$ & & $3.70(1.71-8.04)$ & \\
\hline \multirow[t]{2}{*}{ WBC (/ul) } & $<8000$ & 1093 & $3(4.0 \%)$ & 1 & 0.62 & & \\
\hline & $\geq 8000$ & 76 & $32(2.9 \%)$ & $1.36(0.41-4.56)$ & & & \\
\hline \multirow[t]{2}{*}{ Alb (g/dl) } & $\geq 3.5$ & 1040 & $26(2.5 \%)$ & 1 & 0.0071 & 1 & 0.066 \\
\hline & $<3.5$ & 129 & $9(7.0 \%)$ & $2.92(1.34-6.39)$ & & $2.17(0.95-4.98)$ & \\
\hline \multirow[t]{2}{*}{ CRP (mg/dl) } & $<1$ & 1055 & $29(2.8 \%)$ & 1 & 0.14 & & \\
\hline & $\geq 1$ & 114 & $6(5.3 \%)$ & $1.97(0.80-4.84)$ & & & \\
\hline \multirow[t]{2}{*}{ DM } & Presence & 421 & $6(1.4 \%)$ & 1 & 0.023 & 1 & 0.0056 \\
\hline & Absence & 748 & $29(3.9 \%)$ & $2.79(1.15-6.78)$ & & $3.62(1.46-8.99)$ & \\
\hline \multirow[t]{2}{*}{ Pretreatment $*$} & With & 37 & $1(2.7 \%)$ & 1 & 0.92 & & \\
\hline & Without & 1132 & $34(3.0 \%)$ & $1.11(0.15-8.37)$ & & & \\
\hline \multirow[t]{3}{*}{ Operative procedure } & DP & 406 & $5(1.2 \%)$ & 1 & & 1 & \\
\hline & $\mathrm{PD}$ & 675 & $27(4.0 \%)$ & $3.34(1.28-8.75)$ & 0.014 & $3.06(1.12-8.36)$ & 0.029 \\
\hline & $\mathrm{TP}$ & 88 & $3(3.4 \%)$ & $2.83(0.66-12.1)$ & 0.16 & $3.27(0.73-14.7)$ & 0.12 \\
\hline \multirow[t]{2}{*}{ Operation time (min) } & $<360$ & 597 & $15(2.5 \%)$ & 1 & 0.33 & & \\
\hline & $\geq 360$ & 572 & $20(3.5 \%)$ & $1.41(0.71-2.77)$ & & & \\
\hline \multirow[t]{2}{*}{ Blood loss (ml) } & $<1200$ & 1010 & $26(2.6 \%)$ & 1 & 0.039 & 1 & 0.34 \\
\hline & $\geq 1200$ & 159 & $9(5.7 \%)$ & $2.27(1.04-4.94)$ & & $1.50(0.66-3.43)$ & \\
\hline \multirow[t]{2}{*}{ Vascular resection } & Without & 967 & $29(3.0 \%)$ & 1 & 0.98 & & \\
\hline & With & 202 & $6(3.0 \%)$ & $0.99(0.41-2.42)$ & & & \\
\hline
\end{tabular}

*Pretreatment was preoperative chemotherapy or chemoradiotherapy

**Delayed gastric emptying was defined by the International Study Group on Pancreatic Surgery

$P P H$ postpancreatectomy hemorrhage, $O R$ odds ratio, $C I$ confidence interval, $B M I$ body mass index, WBC white blood cells, $A l b$ albumin, $C R P C$ reactive protein, $D M$ diabetes mellitus, $D P$ distal pancreatectomy, $P D$ pancreatoduodenectomy, $T P$ total pancreatectomy, $D G E$ delayed gastric emptying

\section{Univariate and multivariate analyses of risk factors for POPF after PD (Table 4)}

Because POPF was risk factor for PPH after PD, we assessed risk factors for POPF after PD. In multivariate analysis, BMI $\geq 25 \mathrm{~kg} / \mathrm{m}^{2}$ (OR 3.17), Alb $<3.5 \mathrm{~g} / \mathrm{dl}(\mathrm{OR}$ 1.77), absence of DM (OR 1.75), distal extrahepatic bile duct carcinoma (OR 4.05), and carcinoma of the papilla of Vater (PVC) (OR 5.19) were independent risk factors for POPF after PD.

\section{Association between POPF and PPH after DP and PD}

The incidence of POPF after DP was 19\% (78/406). There was no significant difference in the incidence of POPF between DP and PD [20\% (133/675), $P=0.84]$. Unlike with PD where the OR of 31.7 , there was also no significant association between POPF and PPH after DP [3\% $(2 / 78)$ in patients with POPF and $1 \%(3 / 328)$ in patients without POPF; $P=0.28]$.

\section{Discussion}

This study indicates that absence of DM (HbAlc level $\leq$ $6.2 \%$ ) is an independent risk factor for PPH after all pancreatectomies and PD and for POPF after PD; a risk scoring system including the absence of DM may be useful for predicting PPH before surgery. By determining the value of HbA1c level $(\leq 6.2 \%)$, we can predict PPH more easily. This is a new finding that has not been reported before.

In recent years, PPH has a low frequency, but it has a poor outcome. POPF, DGE, wound infection, and abdominal abscess are major complications of PD [3, 4, 17]. Previous literatures reported that the incidence of PPH was only 3-16\%; however, mortality among patients with PPH was 16-36\% [4-12]. In this study, the incidence and mortality rates of patients with $\mathrm{PPH}$ were 3\% (35/1169) and 11\% (4/35), respectively (Table 1), after all pancreatectomies. PPH was the major cause of death within 30 days after pancreatectomy. Previous literatures also reported that short-term outcomes after pancreatectomy were better in high-volume centers than in low-volume centers (mortality rate $0.9-6.0 \%$ vs. $13.0-18.8 \%)[9,18-20]$. 
Table 3 Univariate and multivariate analyses of risk factors for postpancreatectomy hemorrhage grades B or C after pancreatoduodenectomy

\begin{tabular}{|c|c|c|c|c|c|c|c|}
\hline \multirow[b]{2}{*}{ Risk factors } & \multirow[b]{2}{*}{ Definition } & \multirow[b]{2}{*}{$n$} & \multicolumn{3}{|l|}{ Univariate } & \multicolumn{2}{|l|}{ Multivariate } \\
\hline & & & PPH & $\begin{array}{l}\text { OR } \\
(95 \% \mathrm{CI})\end{array}$ & $P$ value & $\begin{array}{l}\text { OR } \\
(95 \% \mathrm{CI})\end{array}$ & $P$ value \\
\hline \multirow[t]{2}{*}{ Period } & 2005-2011 & 324 & $9(2.8 \%)$ & 1 & 0.12 & & \\
\hline & $2012-2018$ & 351 & $18(5.1 \%)$ & $1.89(0.84-4.27)$ & & & \\
\hline \multirow[t]{2}{*}{ Age (years) } & $<65$ & 251 & $8(3.2 \%)$ & 1 & 0.41 & & \\
\hline & $\geq 65$ & 424 & $19(4.5 \%)$ & $1.43(0.61-3.30)$ & & & \\
\hline \multirow{2}{*}{ Sex } & Female & 264 & $7(2.7 \%)$ & 1 & 0.15 & & \\
\hline & Male & 411 & $20(4.9 \%)$ & $1.88(0.78-4.51)$ & & & \\
\hline \multirow[t]{2}{*}{ BMI $\left(\mathrm{kg} / \mathrm{m}^{2}\right)$} & $<25$ & 595 & $20(3.4 \%)$ & 1 & 0.021 & 1 & 0.14 \\
\hline & $\geq 25$ & 80 & $7(8.8 \%)$ & $2.76(1.13-6.74)$ & & $2.22(0.77-6.43)$ & \\
\hline \multirow[t]{2}{*}{ WBC (/ul) } & $<8000$ & 628 & $25(4.0 \%)$ & 1 & 0.93 & & \\
\hline & $\geq 8000$ & 47 & $2(4.3 \%)$ & $1.07(0.25-4.67)$ & & & \\
\hline \multirow[t]{2}{*}{$\mathrm{Alb}(\mathrm{g} / \mathrm{dl})$} & $\geq 3.5$ & 563 & $17(3.0 \%)$ & 1 & 0.016 & 1 & 0.14 \\
\hline & $<3.5$ & 112 & $10(8.9 \%)$ & $2.68(1.17-6.12)$ & & $2.04(0.79-5.28)$ & \\
\hline \multirow[t]{2}{*}{ CRP (mg/dl) } & $<1$ & 577 & $22(3.8 \%)$ & 1 & 0.56 & & \\
\hline & $\geq 1$ & 98 & $5(5.1 \%)$ & $1.36(0.50-3.67)$ & & & \\
\hline \multirow[t]{2}{*}{ DM } & Presence & 241 & $4(1.7 \%)$ & 1 & 0.021 & 1 & 0.040 \\
\hline & Absence & 434 & $23(5.3 \%)$ & $3.32(1.13-9.70)$ & & $3.45(1.06-11.3)$ & \\
\hline \multirow[t]{2}{*}{ Pre-drainage } & Without & 404 & $13(3.2 \%)$ & 1 & 0.21 & & \\
\hline & With & 271 & $14(5.2 \%)$ & $1.64(0.76-3.54)$ & & & \\
\hline \multirow[t]{2}{*}{ Pretreatment * } & With & 29 & $1(3.5 \%)$ & 1 & 0.87 & & \\
\hline & Without & 646 & $26(4.0 \%)$ & $1.17 ` 0.15-8.97)$ & & & \\
\hline \multirow[t]{2}{*}{ Operation time (min) } & $<360$ & 220 & $9(4.1 \%)$ & $1.04(0.46-2.34)$ & 0.93 & & \\
\hline & $\geq 360$ & 455 & $18(4.0 \%)$ & 1 & & & \\
\hline \multirow{2}{*}{ Blood loss (ml) } & $<1200$ & 566 & $18(3.2 \%)$ & 1 & 0.011 & 1 & 0.27 \\
\hline & $\geq 1200$ & 109 & $9(8.4 \%)$ & $2.81(1.23-6.43)$ & & $1.74(0.65-4.66)$ & \\
\hline \multirow[t]{2}{*}{ Vascular resection } & Without & 566 & $22(3.9 \%)$ & 1 & 0.73 & & \\
\hline & With & 109 & $5(4.6 \%)$ & $1.19(0.44-3.21)$ & & & \\
\hline \multirow{2}{*}{ PJ anastomosis method } & Duct-to mucosa & 580 & $23(4.0 \%)$ & 1 & 0.91 & & \\
\hline & Modified Blumgart & 95 & $4(4.2 \%)$ & $1.06(0.36-3.15)$ & & & \\
\hline \multirow[t]{2}{*}{ Falciform ligament wrapping of GDA stump } & Without & 399 & $16(2.8 \%)$ & 1 & 0.059 & & \\
\hline & With & 276 & $11(5.7 \%)$ & $2.13(0.97-4.66)$ & & & \\
\hline \multirow[t]{2}{*}{ Clipping of GDA stump } & Without & 657 & $26(4.0 \%)$ & 1 & 0.75 & & \\
\hline & With & 18 & $1(5.6 \%)$ & $1.43(0.18-11.1)$ & & & \\
\hline \multirow[t]{2}{*}{ PJ stent } & Without & 318 & $8(2.5 \%)$ & 1 & & & \\
\hline & With & 357 & $19(5.3 \%)$ & $2.18(0.94-5.05)$ & 0.059 & & \\
\hline Pathological tissue type & PDAC & 261 & $6(2.3 \%)$ & 1 & & 1 & \\
\hline & IPMN & 139 & $3(2.2 \%)$ & $0.94(0.23-3.82)$ & 0.93 & $1.13(0.22-5.72)$ & 0.88 \\
\hline & DEBDC & 149 & $12(8.1 \%)$ & $3.74(1.37-10.2)$ & 0.010 & $1.49(0.47-4.75)$ & 0.50 \\
\hline & $\mathrm{PVC}$ & 75 & $4(5.3 \%)$ & $2.40(0.66-8.75)$ & 0.18 & $0.81(0.19-3.49)$ & 0.78 \\
\hline & Others & 50 & $2(4.0 \%)$ & $1.78(0.35-9.07)$ & 0.49 & $2.14(0.34-13.3)$ & 0.42 \\
\hline POPF** & None or A & 542 & $3(0.6 \%)$ & 1 & $<0.0001$ & 1 & $<0.0001$ \\
\hline & $\mathrm{B}$ or $\mathrm{C}$ & 133 & $24(18.1 \%)$ & $39.6(11.8-133.7)$ & & $31.7(8.94-112.2)$ & \\
\hline $\mathrm{DGE}^{* * *}$ & None or A & 626 & $25(4.0 \%)$ & 1 & 0.98 & & \\
\hline & $\mathrm{B}$ or $\mathrm{C}$ & 49 & $2(4.1 \%)$ & $1.02(0.23-4.45)$ & & & \\
\hline Bile leakage & Without & 665 & $26(3.9 \%)$ & 1 & 0.33 & & \\
\hline & With & 10 & $1(10.0 \%)$ & $2.73(0.33-22.4)$ & & & \\
\hline
\end{tabular}

* Pretreatment was preoperative chemotherapy or chemoradiotherapy

**Postoperative pancreatic fistula was defined by the International Study Group on Pancreatic Surgery

***Postoperative delayed gastric emptying was defined by the International Study Group on Pancreatic Surgery

$P P H$ postpancreatectomy hemorrhage, $O R$ odds ratio, $C I$ confidence interval, $B M I$ body mass index, $W B C$ white blood cell, $A l b$ albumin, $C R P$ Creactive protein, $D M$ diabetes mellitus, $G D A$ gastroduodenal artery, $P J$ stent pancreatojejunostomy stent, $P D A C$ pancreatic ductal adenocarcinoma, $D E B D C$ distal extrahepatic bile duct carcinoma, IPMN intraductal papillary mucinous neoplasm, $P V C$ carcinoma of the papilla of Vater, $P O P F$ postoperative pancreatic fistula, $D G E$ delayed gastric emptying

Some previous reports about intraoperative and postoperative factors showed that vascular resection, pancreaticogastrostomy, postoperative bile leakage, postoperative abdominal infection, and especially POPF were independent risk factors for PPH 
Table 4 Univariate and multivariate analyses of preoperative risk factors for postoperative pancreatic fistula grades $\mathrm{B}$ or $\mathrm{C}$ after pancreatoduodenectomy

\begin{tabular}{|c|c|c|c|c|c|c|c|}
\hline \multirow[b]{2}{*}{ Risk factors } & \multirow[b]{2}{*}{ Definition } & \multirow[b]{2}{*}{$n$} & \multicolumn{3}{|l|}{ Univariate } & \multicolumn{2}{|l|}{ Multivariate } \\
\hline & & & POPF & $\begin{array}{l}\text { OR } \\
(95 \% \mathrm{CI})\end{array}$ & $P$ value & $\begin{array}{l}\text { OR } \\
(95 \% \mathrm{CI})\end{array}$ & $P$ value \\
\hline \multirow[t]{2}{*}{ Age (years) } & $<65$ & 251 & $42(16.8 \%)$ & 1 & 0.14 & & \\
\hline & $\geq 65$ & 424 & $91(21.5 \%)$ & $1.36(0.91-2.04)$ & & & \\
\hline \multirow[t]{2}{*}{ Gender } & Female & 264 & $37(14.0 \%)$ & 1 & 0.0024 & 1 & 0.05002 \\
\hline & Male & 411 & $96(23.4 \%)$ & $1.87(1.23-2.83)$ & & $1.57(0.99-2.46)$ & \\
\hline \multirow[t]{2}{*}{ BMI $\left(\mathrm{kg} / \mathrm{m}^{2}\right)$} & $<25$ & 595 & $106(17.8 \%)$ & 1 & 0.0015 & 1 & $<0.0001$ \\
\hline & $\geq 25$ & 80 & $27(33.8 \%)$ & $2.35(1.41-3.91)$ & & $3.17(1.81-5.56)$ & \\
\hline \multirow[t]{2}{*}{ WBC (/ul) } & $<8000$ & 628 & $120(19.1 \%)$ & 1 & 0.16 & & \\
\hline & $\geq 8000$ & 47 & $13(27.7 \%)$ & $1.62(0.83-3.16)$ & & & \\
\hline \multirow[t]{2}{*}{$\mathrm{Alb}(\mathrm{g} / \mathrm{dl})$} & $\geq 3.5$ & 563 & $99(17.6 \%)$ & 1 & 0.0050 & 1 & 0.027 \\
\hline & $<3.5$ & 112 & $34(30.4 \%)$ & $1.93(1.22-3.06)$ & & $1.77(1.07-2.93)$ & \\
\hline \multirow[t]{2}{*}{ CRP (mg/dl) } & $<1$ & 577 & $108(18.7 \%)$ & 1 & 0.12 & & \\
\hline & $\geq 1$ & 98 & $25(25.5 \%)$ & $1.49(0.90-2.45)$ & & & \\
\hline \multirow[t]{2}{*}{ DM } & Presence & 241 & $35(14.5 \%)$ & 1 & 0.0123 & 1 & 0.019 \\
\hline & Absence & 434 & $98(22.6 \%)$ & $1.72(1.12-2.62)$ & & $1.75(1.10-2.78)$ & \\
\hline \multirow[t]{2}{*}{ Pre-drainage } & Without & 404 & $65(16.1 \%)$ & 1 & 0.0042 & 1 & 0.17 \\
\hline & With & 271 & $68(25.1 \%)$ & $1.75(1.19-2.56)$ & & $0.69(0.40-1.17)$ & \\
\hline \multirow[t]{2}{*}{ Pretreatment $*$} & With & 646 & $126(19.5 \%)$ & 1 & 0.54 & & \\
\hline & Without & 29 & $7(24.1 \%)$ & $1.31(0.55-3.14)$ & & & \\
\hline \multirow[t]{5}{*}{ Pathological tissue type } & PDAC & 262 & $30(11.5 \%)$ & 1 & & 1 & \\
\hline & IPMN & 139 & $18(13.0 \%)$ & $1.15(0.62-2.15)$ & 0.66 & $1.14(0.60-2.20)$ & 0.69 \\
\hline & DEBDC & 149 & $49(32.9 \%)$ & $3.79(2.27-6.32)$ & $<0.0001$ & $4.05(2.15-7.62)$ & $<0.0001$ \\
\hline & PVC & 75 & $29(38.7 \%)$ & $4.88(2.67-8.89)$ & $<0.0001$ & $5.19(2.70-9.97)$ & $<0.0001$ \\
\hline & Others & 50 & $7(14.0 \%)$ & $\begin{array}{l}4.80(2.0-8.89) \\
1.26(0.52-3.05)\end{array}$ & 0.61 & $1.05(0.42-2.65)$ & 0.92 \\
\hline
\end{tabular}

*Pretreatment was preoperative chemotherapy or chemoradiotherapy

$P O P F$ postoperative pancreatic fistula, $O R$ odds ratio, $C I$ confidence interval, $B M I$ body mass index, $W B C$ white blood cell, $\mathrm{A} l b$ albumin, $C R P$ c-reactive protein, $D M$ diabetes mellitus, $P J$ stent pancreatojejunostomy stent, $P D A C$ pancreatic ductal adenocarcinoma, $D E B D C$ distal extrahepatic bile duct carcinoma, IPMN intraductal papillary mucinous neoplasm, $P V C$ carcinoma of the papilla of Vater

[5-12]. PPH from a pseudoaneurysm of the GDA stump usually results from POPF $[9,21]$, and the median onset time of PPH was reported as 5-13 days (range $0-58$ days) $[6,10,12]$. In our study, similar results were noted, and it is necessary to pay attention to the onset time from initial surgery (median onset time 22 days, range 0-65 days; Table 1). Previous literatures have mentioned preoperative risk factors for $\mathrm{PPH}$ such as male sex, high BMI, and low Alb level [8, 10, 12, 14]; our study also showed a similar result. Additionally, in our study, "absence of $\mathrm{DM}(\mathrm{HbA}$ lc level $\leq 6.2 \%)$ " was one of the significant independent risk factors for PPH after all pancreatectomies and PD, which has not been reported.

Insulin acts to promote secretion of pancreatic exocrine cells [22]. It has been reported that the pancreatic exocrine function declined as insulin secretion decreased and the exocrine pancreatic glands atrophied [23]. This means that in patients with DM, the pancreatic parenchyma atrophies and becomes hard, and in patients without DM, the pancreatic parenchyma is thick and soft. Traditionally, patients with a soft pancreas, thick parenchyma, and thin pancreatic duct are considered at risk for POPF after PD [24-27]. Pancreatic exocrine function is an important determinant of POPF after PD
[28]. In pancreatic carcinoma, it has been reported that pancreatic parenchyma atrophy is seen from the onset of illness and the main pancreatic duct is dilated [29]. In the same way, in a patient with intraductal papillary mucinous neoplasm, the main pancreatic duct is slightly dilated and the pancreatic parenchyma is relatively decreased and atrophic. In contrast, in patients with distal extrahepatic bile duct carcinoma, main pancreatic duct dilation and pancreatic parenchyma atrophy are rare. Patients with a thick and soft pancreatic parenchyma have a high possibility of POPF and a high risk of PPH after pancreatectomy. In situations where there is no clear definition of the thickness and hardness of the pancreas and it is difficult to judge them preoperatively, the presence or absence of DM (HbAlc level $\leq 6.2 \%$ ) is considered to be one of the good indicators.

Previous literatures reported that omental flaps or grafts around various anastomoses after PD could reduce the incidence of POPF and PPH [30, 31]. In this study, the statistical analysis did not show a significant benefit of wrapping the GDA stump for preventing PPH. We believe we cannot draw sufficient statistical conclusions because of differences in surgical techniques and instruments, differences in applications 
and methods of round ligament wrapping, and the limited use of clips in recent cases. Hence, further clinical evaluation for this is necessary. In addition, pancreatic surgery in patients at high risk for developing PPH may require new precautions to further reduce the incidence of $\mathrm{PPH}$.

\section{Limitations}

Patients from different periods over the 14-year span of the study underwent different diagnostic and treatment modalities owing to the advances in techniques that occurred over time; these variations may have skewed the outcomes of patients treated during the different periods of the study. Moreover, our investigation had a retrospective design and was performed at a single institution; the biases inherent in such settings cannot be completely excluded.

\section{Conclusion}

Male sex, BMI $\geq 25 \mathrm{~kg} / \mathrm{m}^{2}$, absence of DM (HbA1c level $\leq$ $6.2 \%$ ), and PD are independent risk factors for PPH after all pancreatectomies. A risk scoring system including the new preoperative risk factor "absence of DM" would be useful for predicting $\mathrm{PPH}$.

Acknowledgments Wataru Izumo received the Grant for Young Doctors of Abdominal Emergency Clinical Research from the Japanese Society for Abdominal Emergency Medicine (2017-Y-02).

Authors' contributions - Study conception and design of study: W.I. and R.H

- Acquisition of data: All

- Analysis and interpretation of data: W.I. and R.H

- Drafting of manuscript: W.I. and R.H

- Critical revision of manuscript: W.I. and R.H

Funding information This work was supported by the JSPS KAKENHI (grant number 18K08632).

\section{Compliance with ethical standards}

Conflict of interest The authors declare that they have no conflict of interest.

Ethical approval This study was approved by the institutional review board of Tokyo Women's Medical University (approval number 5083) and the Japanese Society for Abdominal Emergency Medicine (approval number 19-2). All procedures involving human participants were conducted in accordance with the ethical standards of the institutional research committee and with the 1964 Helsinki Declaration. This article does not contain any studies with animals performed by any of the authors.

Informed consent Informed consent was obtained from all individual participants included in the study.
Open Access This article is distributed under the terms of the Creative Commons Attribution 4.0 International License (http:// creativecommons.org/licenses/by/4.0/), which permits unrestricted use, distribution, and reproduction in any medium, provided you give appropriate credit to the original author(s) and the source, provide a link to the Creative Commons license, and indicate if changes were made.

\section{References}

1. Kimura W (2008) Strategies for the treatment of invasive ductal carcinoma of the pancreas and how to achieve zero mortality for pancreaticoduodenectomy. J Hepato-Biliary-Pancreat Surg 15(3): 270-277

2. Nimura $Y$, Nagino M, Takao S, Takada T, Miyazaki K, Kawarada $Y$ et al (2012) Standard versus extended lymphadenectomy in radical pancreatoduodenectomy for ductal adenocarcinoma of the head of the pancreas: long-term results of a Japanese multicenter randomized controlled trial. J Hepatobiliary Pancreat Sci 19(3):230-241

3. Kimura W, Miyata H, Gotoh M, Hirai I, Kenjo A, Kitagawa Y et al (2014) A pancreaticoduodenectomy risk model derived from 8575 cases from a national single-race population (Japanese) using a web-based data entry system: the 30-day and in-hospital mortality rates for pancreaticoduodenectomy. Ann Surg 259(4):773-780

4. Balcom JH, Rattner DW, Warshaw AL, Chang Y, Fernandez-del CC (2001)Ten-year experience with 733 pancreatic resections: changing indications, older patients, and decreasing length of hospitalization. Arch Surg 136(4):391-398

5. Yekebas EF, Wolfram L, Cataldegirmen G, Habermann CR, Bogoevski D, Koenig AM et al (2007) Postpancreatectomy hemorrhage: diagnosis and treatment: an analysis in 1669 consecutive pancreatic resections. Ann Surg 246(2):269-280

6. Darnis B, Lebeau R, Chopin-Laly X, Adham M (2013) Postpancreatectomy hemorrhage (PPH): predictors and management from a prospective database. Langenbeck's Arch Surg 398: $441-448$

7. Roulin D, Cerantola Y, Demartines N, Schäfer M (2011) Systematic review of delayed postoperative hemorrhage after pancreatic resection. J Gastrointest Surg 15:1055-1062

8. Wellner UF, Kulemann B, Lapshyn H, Hoeppner J, Sick O, Makowiec F et al (2014) Postpancreatectomy hemorrhage-incidence, treatment, and risk factors in over 1,000 pancreatic resections. J Gastrointest Surg 18(3):464-475

9. Grützmann R, Rückert F, Hippe-Davies N, Distler M, Saeger HD(2012) Evaluation of the International Study Group of Pancreatic Surgery definition of post-pancreatectomy hemorrhage in a highvolume center. Surgery 151:612-620

10. Feng J, Chen YL, Dong JH, Chen MY, Cai SW, Huang ZQ (2014)Post-pancreaticoduodenectomy hemorrhage: risk factors, managements and outcomes. Hepatobiliary Pancreat Dis Int 13(5):513-522

11. Gao Q-X, Lee H-Y, Wu W-H, Gao S, Yang Y-M, Ma IT et al (2012) Factors associated with post-pancreaticoduodenectomy hemorrhage: 303 consecutive cases analysis. Chin Med J 125:1571-1575

12. Gao F, Li J, Quan S, Li F, Ma D, Yao L et al (2016) Risk factors and treatment for hemorrhage after pancreaticoduodenectomy: a case series of 423 patients. Biomed Res Int 2016:2815693

13. Wente MN, Veit JA, Bassi C, Dervenis C, Fingerhut A, Gouma DJ et al (2007) Postpancreatectomy hemorrhage (PPH): an International Study Group of Pancreatic Surgery (ISGPS) definition. Surgery. 142(1):20-25

14. Wellner UF, Makowiec F, Sick O, Hopt UT, Keck T (2012) Arguments for an individualized closure of the pancreatic remnant 
after distal pancreatic resection. World J Gastrointest Surg 4(5): 114-120

15. Bassi C, Dervenis C, Butturini G, Fingerhut A, Yeo C, Izbicki J et al (2005) International Study Group on Pancreatic Fistula Definition. Postoperative pancreatic fistula: an international study group (ISGPF) definition. Surgery 138(1):8-13

16. Wente MN, Bassi C, Dervenis C, Fingerhut A, Gouma DJ, Izbicki JR et al (2007) Delayed gastric emptying (DGE) after pancreatic surgery: a suggested definition by the International Study Group of Pancreatic Surgery (ISGPS). Surgery 142(5):761-768

17. Yeo CJ, Cameron JL, Sohn TA, Lillemoe KD, Pitt HA, Talamini MA et al (1997) Six hundred fifty consecutive pancreaticoduodenectomies in the 1990s: pathology, complications, and outcomes. Ann Surg 226(3):248-257 discussion 257-60

18. Gordon TA, Burleyson GP, Tielsch JM, Cameron JL (1995) The effects of regionalization on cost and outcome for one general highrisk surgical procedure. Ann Surg 221(1):43-49

19. Sosa JA, Bowman HM, Gordon TA, Bass EB, Yeo CJ, Lillemoe $\mathrm{KD}$ et al (1998) Importance of hospital volume in the overall management of pancreatic cancer. Ann Surg 228:429-438

20. Lieberman MD, Kilburn H, Lindsey M, Brennan MF (1995) Relation of perioperative deaths to hospital volume among patients undergoing pancreatic resection for malignancy. Ann Surg 222: 638-645

21. Fuks D, Piessen G, Huet E, Tavernier M, Zerbib P, Michot F et al (2009)Life-threatening postoperative pancreatic fistula (grade C) after pancreaticoduodenectomy: incidence, prognosis, and risk factors. Am J Surg 197:702-709

22. Williams JA, Goldfine ID (1985) The insulin-pancreatic acinar axis. Diabetes 34(10):980-986

23. Adler G, Kern HF (1975) Regulation of exocrine pancreatic secretory process by insulin in vivo. Horm Metab Res 7(4):290-296

24. Sugimoto M, Takahashi S, Kojima M, Kobayashi T, Gotohda N, Konishi M (2017) In patients with a soft pancreas, a thick parenchyma, a small duct, and fatty infiltration are significant risks for pancreatic fistula after pancreaticoduodenectomy. J Gastrointest Surg 21(5):846-854

25. Kawai M, Kondo S, Yamaue H, Wada K, Sano K, Motoi F et al (2011) Predictive risk factors for clinically-relevant pancreatic fistula analyzed in 1,239 patients with pancreaticoduodenectomy: multicenter data collection as a project study of pancreatic surgery by the Japanese Society of HepatoBiliary-Pancreatic Surgery. J Hepatobiliary Pancreat Sci 18:601-608

26. Ansorge C, Strömmer L, Andrén-Sandberg Å, Lundell L, Herrington MK, Segersvärd R (2012) Structured intraoperative assessment of pancreatic gland characteristics in predicting complications after pancreaticoduodenectomy. Br J Surg 99:1076-1082

27. El Nakeeb A, Salah T, Sultan A, El Hemaly M, Askr W, Ezzat H et al (2013) Pancreatic anastomotic leakage after pancreaticoduodenectomy. Risk factors, clinical predictors, and management (single center experience). World J Surg 37:14051418

28. Hamanaka Y, Nishihara K, Hamasaki T, Kawabata A, Yamamoto S, Tsurumi M et al (1996) Pancreatic juice output after pancreatoduodenectomy in relation to pancreatic consistency, duct size, and leakage. Surgery 119(3):281-287

29. Masamune A, Shimosegawa T. Pancreatic stellate cells: a dynamic player of the intercellular communication in pancreatic cancer. Clin Res Hepatol Gastroenterol 2015; 39 (Suppl 1): S98-103

30. Seyama Y, Kubota K, Kobayashi T, Hirata Y, Itoh A, Makuuchi M (1998)Two-staged pancreatoduodenectomy with external drainage of pancreatic juice and omental graft technique. J Am Coll Surg 187(1):103-105

31. Shah OJ, Bangri SA, Singh M, Lattoo RA, Bhat MY (2015) Omental flaps reduces complications after pancreaticoduodenectomy. Hepatobiliary Pancreat Dis Int 14(3):313-319

Publisher's note Springer Nature remains neutral with regard to jurisdictional claims in published maps and institutional affiliations. 